\title{
A Joint TDOA-PDOA Localization Approach Using Particle Swarm Optimization
}

\author{
Hui Chen, Student Member, IEEE, Tarig Ballal, Member, IEEE, Nasir Saeed, Senior Member, IEEE, \\ Mohamed-Slim Alouini, Fellow, IEEE and Tareq Y. Al-Naffouri, Senior Member, IEEE
}

\begin{abstract}
Estimating the location of a target is essential for many applications such as asset tracking, navigation, and data communications. Time-difference-of-arrival (TDOA) based localization has the main advantage that it does not require synchronization between the transmitting and the receiving sides. Phase-difference-of-arrival (PDOA) provides additional information that can be leveraged to enhance localization performance. The combination of TDOA and PDOA for localization has not been reported in the literature. In this paper, we propose a novel approach that incorporates both TDOA and PDOA to achieve improved position estimation. In the proposed approach, an initial location estimate is obtained by optimizing a TDOA cost function. Next, a PDOA, or a hybrid TDOA-PDOA cost function is optimized using a particle swarm optimizer to obtain the final location estimate. Simulation results show that the proposed approach sufficiently, and justifiably, improves localization performance relative to pure TDOA methods.
\end{abstract}

Index Terms-Localization, TDOA, PDOA, particle swarm optimizer

\section{INTRODUCTION}

Existing and future wireless communication technologies require accurate real-time localization and tracking methods. Location information is important for numerous applications such as target monitoring [1], wireless sensor network [2], navigation [3], drones localization [4] and connected vehicles [5]. A survey of positioning techniques and systems can be found in [6], [7].

Time-difference-of-arrival (TDOA) based localization algorithms [8] are mainly used because no synchronization is required between the target and the anchors. The anchors share the same timing so that the TDOA information of the signal between different anchors can be estimated, and hence the location can be obtained. There are two main types of methods to solve the nonlinear localization problems using the TDOA measurements. One solution is linearization, followed by a least-squares (LS) estimation. Despite the obvious advantage of linearization in simplifying these algorithms, the same linearization operation makes these algorithms more sensitive to noise [9], [10]. Formulating the localization task as a nonconvex optimization problem is another way that can be solved by using, for example, iterative optimization methods [11], [12], or convex relaxation methods [13], [14].

PDOA information is customarily used in far-field scenarios where anchor separations are small [15], [16]. For large

The authors are with the Division of Computer, Electrical and Mathematical Science \& Engineering, King Abdullah University of Science and Technology (KAUST), Thuwal, 23955-6900, KSA. e-mail: (\{hui.chen; tarig.ahmed; nasir.saeed; slim.alouini; tareq.alnaffouri $\} @$ kaust.edu.sa). inter-anchor distance, the actual phase difference of a signal between a pair of anchors cannot be computed in a direct way. This is due to the phase wrapping problem [15], which results in an observed wrapped phase difference that cannot be directly used. Despite the phase wrapping issue, PDOA provides high measurement precision compared to TDOA [15], [17]-[19].

To leverage the high precision of PDOA, we propose a localization technique that combines both TDOA and PDOA measurements. We consider two problem formulations. In the first formulation, we use only PDOA measurements, whereas in the second formulation, we consider a hybrid cost function using both TDOA and PDOA information. In both cases, the resulting cost functions suffer from the presence of large numbers of local minima. To obtain a feasible solution, we utilize a particle swarm optimizer (PSO) [20] to solve the two optimization problems. A PSO is a multidimensional optimization technique inspired by the behavior of bird flock searching for food [21]. It has the advantages of simple implementation, high-quality solutions to global optima, and quick convergence [22]. For the PSO to work well, the optimizer needs to start from a good initial guess of the target's location and to have sufficient swarms to search for a global optimum. To provide such a good initialization, we rely on the pure TDOA approach whose cost function is much easier to optimize than our proposed cost functions.

Numerical simulations were carried out to evaluate the performance of the proposed joint TODA-PDOA localization method using PSO. The proposed method is compared to pure TDOA localization implemented using a linear closedform, and using a semidefinite programming (SDP) convex relaxation optimization method.

The remainder of this paper is organized as follows. Section II presents the proposed joint TDOA-PDOA localization algorithm. Section III discusses simulation setup and presents the results, while Section IV, states the conclusion of the paper.

\section{The Proposed Localization Algorithm}

\section{A. The TDOA Approach}

Consider a 2-D or 3-D space with a system of $N$ anchors $R_{1}, R_{2}, \ldots, R_{N}$ located at $\mathbf{r}_{i}, i=1, \ldots, N$, and a target located at a point $\mathbf{p}$. For any signal, the time difference of arrival between anchor $R_{i}$ and anchor $R_{j}$ at point $\mathbf{p}$ is given by

$$
\tau_{i j}(\mathbf{p})=\frac{\left\|\mathbf{p}-\mathbf{r}_{i}\right\|_{2}-\left\|\mathbf{p}-\mathbf{r}_{j}\right\|_{2}}{v}, i, j=1, \ldots, N,
$$


where $\|\cdot\|_{2}$ denotes the $L_{2}$ norm, and $v$ is the signal propagation speed. This formula gives the exact (true) TDOA. Practically, we have to rely on estimates, $\hat{\tau}_{i j}$, of TDOA. With time synchronization maintained between receivers, TDOA can be estimated using one of two approaches. By transferring the received signals to a central processor, a cross-correlation based method can be used to obtain TDOA estimates [8]. An alternative approach is based on estimating the times of arrival of signal at each anchor independently. The differences of these times give the required TDOA estimates [13].

With TDOA information acquired through any of the aforementioned or other methods, the target location can be estimated. Among the candidate methods are the linear closed-form method [9] where a linearization is performed to simplify the problem, which results in exacerbating the noise effect. Other techniques are based on combining weighted least squares and firefly algorithm [23], and $\ell_{1}$ minimization [24]. In this paper, we adopt a maximum likelihood approach which can be pursued as follows [13]:

$$
\hat{\mathbf{p}}=\underset{\mathbf{p}}{\arg \min } c_{t}(\mathbf{p})=\underset{\mathbf{p}}{\arg \min } \sum_{i=1}^{N} \sum_{\substack{j=1 \\ j \neq i}}^{N}\left[\hat{\tau}_{i j}-\tau_{i j}(\mathbf{p})\right]^{2} .
$$

To have an idea of the properties of the function $c_{t}(\mathbf{p})$ in (2), we expand its expression using (1) to obtain

$$
\begin{aligned}
c_{t}(\mathbf{p}) & =\sum_{i=1}^{N} \sum_{\substack{j=1 \\
j \neq i}}^{N} \frac{1}{v^{2}}\left\|\mathbf{p}-\mathbf{r}_{i}\right\|_{2}^{2}+\frac{1}{v^{2}}\left\|\mathbf{p}-\mathbf{r}_{j}\right\|_{2}^{2} \\
& -\frac{2}{v^{2}}\left\|\mathbf{p}-\mathbf{r}_{i}\right\|_{2}\left\|\mathbf{p}-\mathbf{r}_{j}\right\|_{2}-\frac{2 \hat{\tau}_{i j}}{v}\left\|\mathbf{p}-\mathbf{r}_{i}\right\|_{2} \\
& +\frac{2 \hat{\tau}_{i j}}{v}\left\|\mathbf{p}-\mathbf{r}_{j}\right\|_{2}+\hat{\tau}_{i j}^{2} .
\end{aligned}
$$

Based on (3), we can see that $c_{t}(\mathbf{p})$ can be convex (only) for certain choices of $\hat{\tau}_{i j}$. However, in the general case, the function $c_{t}(\mathbf{p})$ has to be dealt with in the non-convex optimization framework. To this end, convex relaxation [13], [14] represents one of the effective tools.

An example of $c_{t}(\mathbf{p})$ for a target at $[0,0]^{T} \mathrm{~m}$, with the target and anchors all lying in the plane $z=2$, is shown in Fig. 1 (a-1, a-2). A global minimum can be observed at $[0,0]^{T}$, the true target location. It is observed that the function is relatively flat around the global minimum, which makes the localization accuracy more susceptible to TDOA estimation errors. Existing methods for TDOA estimation are limited in their accuracy by the effect of noise, the sampling rate, time resolution and other factors [15], [17]-[19]. To enhance localization accuracy, our proposed approach leverages, in addition to TDOA, the more accurate estimates of PDOA.

\section{B. The proposed PDOA Cost Function}

Given signal frequencies $\mathbf{f}=\left[f_{1}, \ldots, f_{M}\right]^{T}$, PDOA is related to TDOA through

$$
\phi_{i j, m}(\mathbf{p})=2 \pi f_{m} \tau_{i j}(\mathbf{p}) .
$$

Here, $\phi_{i j, m}$ is the PDOA between the received signals at anchors $R_{i}$ and $R_{j}$ at frequency $f_{m}$. Due to the way PDOA is

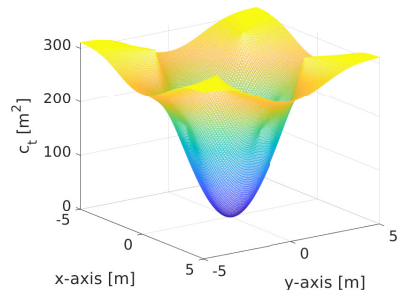

(a-1) $c_{t}(\mathbf{p})$

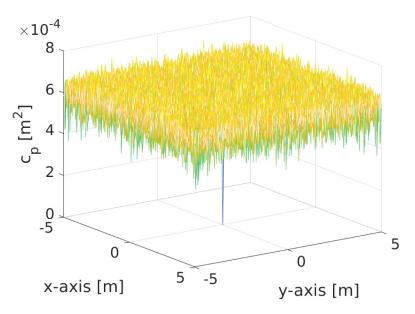

(b-1) $c_{p}(\mathbf{p})$

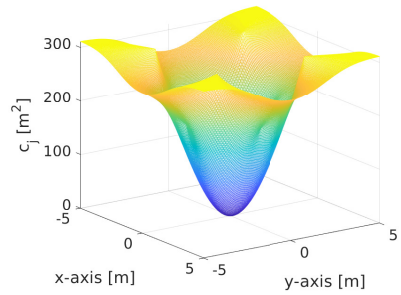

(c-1) $c_{j}(\mathbf{p})$

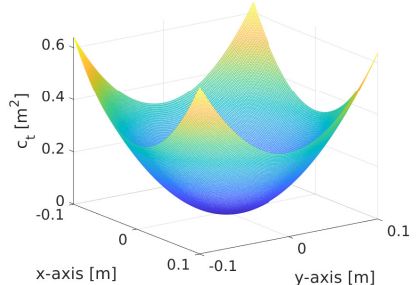

(a-2) $c_{t}(\mathbf{p})$

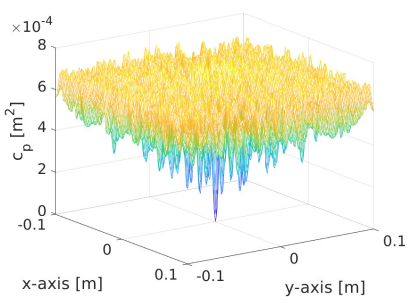

(b-2) $c_{p}(\mathbf{p})$

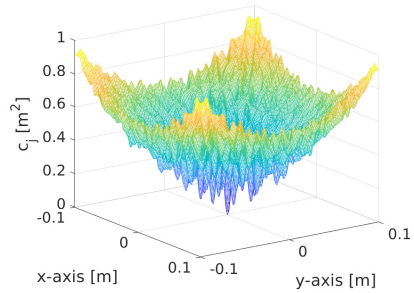

(c-2) $c_{j}(\mathbf{p})$
Fig. 1. Example plots of the TDOA, PDOA, and the joint cost functions. The second column plots are obtained by zooming in around the global minimum.

usually estimated, mostly, we only have access to a wrapped version of it that is given by [25]

$$
\psi_{i j, m}(\mathbf{p})=\operatorname{wrap}\left(\phi_{i j, m}(\mathbf{p})\right)=\bmod \left(\phi_{i j, m}(p)+\pi, 2 \pi\right)-\pi
$$

where $\psi_{i j, m} \in[-\pi, \pi)$ is the wrapped PDOA, and $\bmod (a, b)$ returns the remainder of dividing $a$ by $b$.

Practically, the PDOA of two signals can be estimated directly from the cross power spectrum of the signals [15], [25]. This method requires the two received signals to be transferred to a central processor. Another method to estimate the PDOA is by recording the phase of arrival of the signal at each anchor independently and subtracting the two phases to obtain the PDOA [18], [19]. The method proposed in this paper does not depend on how the PDOA is obtained.

Given the estimated (wrapped) PDOA, we create the following cost function:

$$
c_{p}(\mathbf{p})=\frac{1}{M} \sum_{m=1}^{M} b_{m} \sum_{i=1}^{N} \sum_{\substack{j=1 \\ j \neq i}}^{N}\left\{\operatorname{wrap}\left[\hat{\psi}_{i j, m}-\psi_{i j, m}(\mathbf{p})\right]\right\}^{2} .
$$

In (6), the wrapped PDOA $\psi_{i j, m}(\mathbf{p})$ can be computed using (5). The scalars $b_{m}$ are weighting coefficients given by

$$
b_{m}=\frac{\sigma_{t}^{2}}{\sigma_{p, m}^{2}}=\frac{\sigma_{t}^{2}}{\gamma_{p, m}^{2}}\left(2 \pi f_{m}\right)^{2} ;
$$


where $\sigma_{t}^{2}$ and $\sigma_{p, m}^{2}$ are the TDOA and PDOA estimation error variances in squared second; and $\gamma_{p, m}^{2}$ are PDOA variances given in squared radians. Practical ways of estimating the error variances of TDOA and PDOA are using the SNRs [10], [25]. Note that the inclusion of $\sigma_{t}^{2}$ in the weights $b_{m}$ has no effect on the locations of the minima of $c_{p}(\mathbf{p})$. The purpose of scaling the weights by this factor will be explained in the next subsection.

The function $c_{p}(\mathbf{p})$ is non-convex (at least) due to the nonlinearity of the wrapping operation. Since wrapping occurs in a periodic fashion at an interval of $2 \pi$, we expect $c_{p}(\mathbf{p})$ to be highly non-convex with many local minima. The purpose of the wrap operation in (6) is to allow us to compute the residual errors on a circle rather than computing them on a line. For example, when $\hat{\psi}=\pi-\epsilon, \psi=-\pi+\epsilon$, and $0<\epsilon<\pi / 2$, we have $\hat{\psi}-\psi=2 \pi-2 \epsilon$ and $\operatorname{wrap}(\hat{\psi}-\psi)=-2 \epsilon$. The latter result show that the two PDOA values are closer than what their difference indicates. An example visualization of $c_{p}(\mathbf{p})$ is shown in Fig. 1 $(\mathbf{b}-1, \mathbf{b}-2)$ for the same setup used in Fig. 1 (a-1, a-2).

Remark 1: The proposed PDOA cost function in (6) can be related to the TDOA cost function in (2). From the definition of the wrap function, it is to easy to see that

$$
\begin{aligned}
\operatorname{wrap}\left[\hat{\psi}_{i j, m}-\psi_{i j, m}(\mathbf{p})\right] & =\operatorname{wrap}\left[\hat{\phi}_{i j, m}-\phi_{i j, m}(\mathbf{p})\right] \\
& =\operatorname{wrap}\left\{2 \pi f_{m}\left[\hat{\tau}_{i j, m}-\tau_{i j, m}(\mathbf{p})\right]\right\} .
\end{aligned}
$$

Based on (8), it can be said that (6) represents another form of TDOA localization where the residuals undergo a nonlinear transformation through the wrap function. This transformation is not unique, which makes the cost function difficult to optimize. Note that the main merit of (6) is based on the relatively high quality of the PDOA measurements, and by extension, the corresponding TDOA estimates.

\section{The Proposed Joint Cost Function}

One can consider combining both TDOA and PDOA measurements to form a joint cost function that leverages all the available information. We propose a simple way to achieve this by using a composite function given by

$$
c_{j}(\mathbf{p})=c_{t}(\mathbf{p})+c_{p}(\mathbf{p}),
$$

where $c_{t}(\mathbf{p})$ and $c_{p}(\mathbf{p})$ are defined in (3) and (6), respectively. It is important to note here that the cost function $c_{j}(\mathbf{p})$ does not combine the contributions of TDOA and PDOA equally. Recalling (6) and (7), we observe that $c_{p}(\mathbf{p})$ is already weighted by the TDOA error variance $\sigma_{t}^{2}$. In addition, the contributions of different frequencies to $c_{p}(\mathbf{p})$ are weighted by the inverse of the corresponding PDOA error variances. The implication of this weighing is to emphasize the contribution from the more precise measurements, TDOA or PDOA.

The joint function in $c_{j}(\mathbf{p})$ is non-convex in $\mathbf{p}$ by definition. It is also expected to inherit some of the characteristics of the cost function $c_{p}(\mathbf{p})$, especially the large number of local minima. An example plot of $c_{j}(\mathbf{p})$ is shown in Fig. 11 (c-1, c-2) which corresponds to the same scenario depicted in Fig. 1 (a-1, a-2).

\section{Optimization}

In this paper, we consider localization based on both cost functions (6) and (9). The target location can be estimated by finding the global minimum by solving

$$
\hat{\mathbf{p}}_{p}=\underset{\mathbf{p}}{\arg \min } c_{p}(\mathbf{p}),
$$

or

$$
\hat{\mathbf{p}}_{j}=\underset{\mathbf{p}}{\arg \min } c_{j}(\mathbf{p}) .
$$

To avoid picking up a local minimum as the final result, a PSO (particle swarm optimizer) is used [20], [22]. PSO initializes a set of candidate solutions (swarm size $s$ ) around an initial point $\mathbf{p}_{0}$. However, it is not always guaranteed for the PSO to find the global minimum as the swarm size and searching area decide the performance. Two key processes to achieve good performance are 1) to judiciously set the initial point for the PSO algorithm, and 2) to set the boundaries of the search space.

To provide a good initial point to the PSO, we rely on solving the classical (pure) TDOA problem (2) as a means to acquire a good initialization point. A plethora of methods, with well-understood performance characteristics, exist to achieve this task. Namely, we utilize a quasi-Newton optimizer to solve the optimization problem (2) and initialize the PSO algorithm.

We define the search space of the PSO as

$$
\left\{\begin{array}{c}
S_{l b}=\max \left(\mathbf{p}_{0}-\delta, \mathbf{p}_{l b}\right) \\
S_{u b}=\min \left(\mathbf{p}_{0}+\delta, \mathbf{p}_{u b}\right)
\end{array},\right.
$$

where $\mathbf{p}_{l b}, \mathbf{p}_{u b}$ are the lower and upper bound of the space covered by the anchors, and $\delta$ is a vector of the same dimensionality as $\mathbf{p}_{0}$. We propose the following choice of the parameter $\delta$ :

$$
\delta=\alpha \sigma_{t} \mathbf{1}
$$

where $\sigma_{t}$ is the standard deviation of the TDOA error, $\mathbf{1}$ is an all-ones vector, and $\alpha$ is a scaling factor. We use $\alpha=0.8$ in all our tests.

By using (12) and (13), we can set the boundaries of the search space of the PSO. Defining the boundaries this way improves the chances of the solutions of (10) and (11) to converge to global optima while reducing the overall computational complexity.

\section{E. Summary of the Proposed TDOA-PDOA Localization Ap- proach}

i Using TDOA measurements, calculate the initial location by solving (2) using a quasi-Newton optimizer [12].

ii Define the lower bound, $S_{l b}$, and the upper bound, $S_{u b}$, of the search space using (12) and (13).

iii Algorithm 1 (TDOA-PDOA-1): Perform (i) and (ii); then solve (10) using PSO.

iv Algorithm 2 (TDOA-PDOA-2): Perform (i) and (ii); then solve (11) using PSO.

Note that, for Algorithm 1, despite the absence of TDOA from the expression of the cost function, it is still essential to use TDOA information for successful initialization of this algorithm. 


\section{Simulations}

\section{A. Simulation Setup}

To simplify the presentation of the results, we use a specific anchor configuration with $N=8$ receivers to test our proposed method. The receivers are located at $[-d,-d],[-d, 0],[-d, d]$, $[0,-d],[0, d],[d,-d],[d, 0],[d, d]$ forming a $2 d \times 2 d \mathrm{~m}^{2}$ square. In all simulations, $d=2.5$ meters is used.

For simplicity, we consider a 2-D localization problem where the target is on the same plane as the anchors. All the simulation results presented in the following subsection are obtained for a target at $[1,1]^{T}$ (all coordinates measured in meters). TDOAs are calculated using (1), while wrapped PDOAs are calculated based on (5) for a single acoustic frequency. $f=21 \mathrm{kHz}$ (e.g., $M=1$ ). The results, however, can easily be extended to the multi-frequency/multi-carrier case. TDOA and PDOA errors are simulated as Gaussian noises with zero mean and standard deviations $\sigma_{t}$ and $\sigma_{p}$, respectively. We set $\sigma_{p, m}=\sigma_{p}, \forall m \in\{1, \cdots, M\}$. The root mean squared error (RMSE) is adopted as the performance metric. For each simulation case, the RMSE is calculated from 5000 trials.

\section{B. Results}

The proposed approach yields two algorithms, TDOAPDOA-1 and TDOA-PDOA-2, which are summarized in Section II-E The performance of the proposed approach is compared to that of the linear closed-form method (TDOALinear) [9], the SDP relaxation method (TDOA-SDP) [13] and the iterative optimization method (TDOA-Iter) [12]. The CRLB for TDOA-based localization (CRLB-T), lower bound for PDOA-based localization (LB-P) [26] and the joint TDOAPDOA lower bound (LB-J) [26] are also used to compare performance.

The simulation results are shown in Fig. 2 to Fig. 5 We can see from the figure that TDOA-Iter performs the best amongst the pure TDOA-based localization algorithm. The proposed TDOA-PDOA methods provide superior results which stay close to the LB in many cases.

In Fig. 2. we study the impact of the number of PSO swarms on the performance of the two proposed algorithms. The setup considers a TDOA error standard deviation $\sigma_{t}=2 \mathrm{~mm}$ (normalized by multiplying the signal propagation speed), a PDOA error standard deviation $\sigma_{p}=0.32 \mathrm{~mm}(0.1265 \mathrm{rad})$, and a search space of size $40 \times 40 \mathrm{~mm}^{2}$. The RMSE of the proposed algorithms tends to converge to the lower bound as the swarm size increases. This means a high probability to find the global minimum. However, with the help of TDOA information, TDOA-PDOA-2 requires less swarms to reach the lower bound. The results in Fig. 2 shows a significant advantage using our proposed joint method in location estimation.

Fig. 3 plots the RMSE against the TDOA error standard deviation $\sigma_{t}$ for $\sigma_{p}=0.32 \mathrm{~mm}$ and $s=500$ swarms. When $\sigma_{t}$ is small compared to $\sigma_{p}$, the joint lower bound LB-J is close to the TDOA CRLB. On the contrary, when $\sigma_{t}$ is relatively large, LB-J is closer to LB-P. However, when $\sigma_{t}$ is excessively large, an erroneous TDOA position will not facilitate the initialization of the proposed algorithm. Fig. 3 emphasizes that,

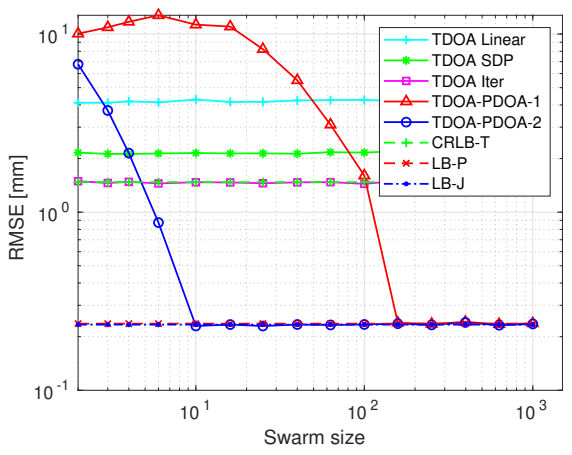

Fig. 2. Performance versus number of swarms $s$ for $\sigma_{t}=2 \mathrm{~mm}$ and $\sigma_{p}=$ $0.32 \mathrm{~mm}$.

for sufficiently small $\sigma_{p}$, the proposed approach yield results with RMSE close to the lower bounds.

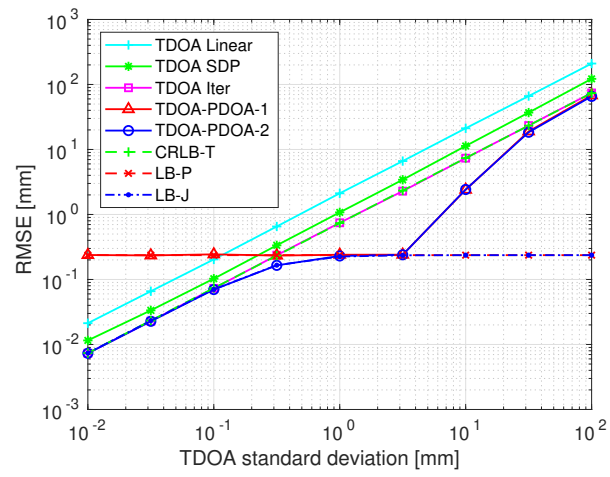

Fig. 3. Performance versus TDOA error standard deviation $\sigma_{t}$ for $\sigma_{p}=$ $0.32 \mathrm{~mm}$ and $s=500$ swarms.

In Fig. 4, we vary the PDOA error standard deviation $\sigma_{p}$, while fixing $\sigma_{t}=5 \mathrm{~mm}$ and $s=500$ swarms. When $\sigma_{p}$ is large, with an accurate initial position estimation, TDOAPDOA-2 has the performance close to the CRLB-T. However, TDOA-PDOA-1 has a worse performance because the TDOA information is not utilized in the optimization procedure. TDOA-PDOA-2 outperforms the rest of the methods, which highlights the benefit of the joint cost function 9 .

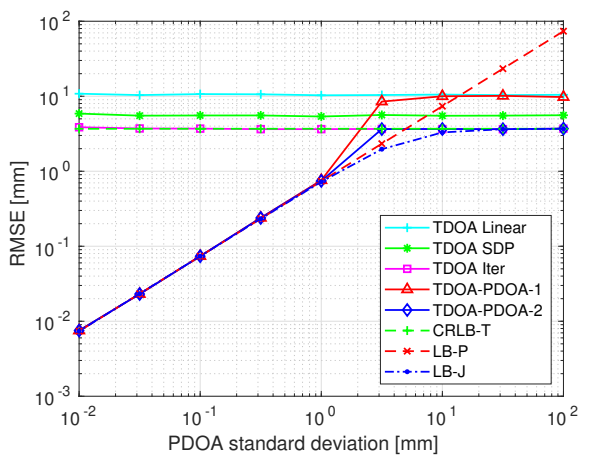

Fig. 4. Performance versus PDOA error standard deviation $\sigma_{p}$ for $\sigma_{t}=$ $50 \mathrm{~mm}$ and $s=500$ swarms.

In Fig. 5, we vary both $\sigma_{t}$ and $\sigma_{p}$ while maintaining 
$\sigma_{p}=\sigma_{t} / 2$. When $\sigma_{t}$ is small, both TDOA-PDOA-1 and TDOA-PDOA-2 can achieve the lower bounds LB-P,and LB-J, respectively. This is because of the accurate initial position and the relatively small search space. With the increase of noise level, the performance of the proposed methods deteriorate because the fixed swarm size used is not sufficient to find the global minimum within a large search area.

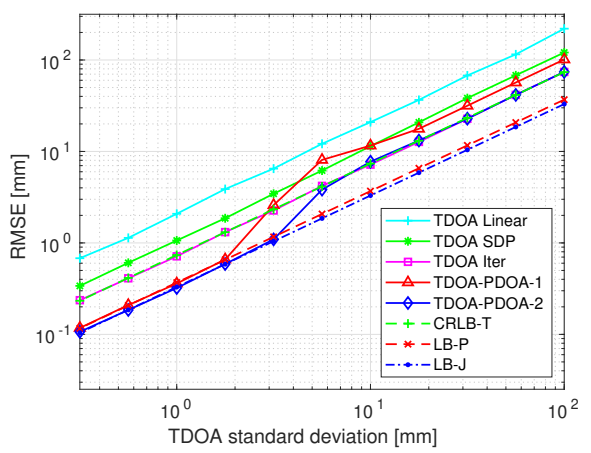

Fig. 5. Performance versus variable TDOA and PDOA error standard deviations, $\sigma_{p}=\sigma_{t} / 2$, and $s=500$ swarms.

\section{CONCLUSION}

A localization approach that jointly uses time-differenceof-arrival (TDOA) and phase-difference-of-arrival (PDOA) measurements has been presented. Two cost functions were proposed; one using only PDOA information, and the other using a joint function that takes advantage of both TDOA and PDOA measurements. A particle swarm optimizer (PSO) is employed to minimize the proposed cost functions and obtain the final location of the target. The initialization of the PSO is facilitated by TDOA-based location estimations. Numerical results show that the proposed methods clearly outperform benchmark methods that do not leverage PDOA information. The proposed methods also stay close to the estimation lower bound for moderate SNR values. The performance of the proposed approach depends on the TDOA estimation result, and the PSO swarm size which dictates the computational complexity of the proposed approach. As demonstrated by simulation results, the benefit of the proposed approach can be attained using moderate swarm sizes.

\section{REFERENCES}

[1] Y. Shu, Y. Huang, J. Zhang, P. Coué, P. Cheng, J. Chen, and K. G. Shin, "Gradient-based fingerprinting for indoor localization and tracking," IEEE Transactions on Industrial Electronics, vol. 63, no. 4, pp. 2424 2433, 2015.

[2] C.-H. Ou, "A localization scheme for wireless sensor networks using mobile anchors with directional antennas," IEEE Sensors Journal, vol. 11, no. 7, pp. 1607-1616, 2011.

[3] M. Murata, D. Ahmetovic, D. Sato, H. Takagi, K. M. Kitani, and C. Asakawa, "Smartphone-based indoor localization for blind navigation across building complexes," in IEEE International Conference on Pervasive Computing and Communications (PerCom), 2018, pp. 1-10.

[4] G. Yang, X. Shi, L. Feng, S. He, Z. Shi, and J. Chen, "CEDAR: A cost-effective crowdsensing system for detecting and localizing drones," IEEE Transactions on Mobile Computing, 2019.
[5] M. Shen, J. Sun, H. Peng, and D. Zhao, "Improving localization accuracy in connected vehicle networks using Rao-Blackwellized particle filters: Theory, simulations, and experiments," IEEE Transactions on Intelligent Transportation Systems, vol. 20, no. 6, pp. 2255-2266, 2018.

[6] X. Guo, N. Ansari, F. Hu, Y. Shao, R. Nkrow, and L. Li, "A survey on fusion-based indoor positioning," IEEE Communications Surveys \& Tutorials, 2019.

[7] N. Saeed, H. Nam, T. Y. Al-Naffouri, and M.-S. Alouini, "A state-of-theart survey on multidimensional scaling based localization techniques," IEEE Communications Surveys Tutorials, pp. 1-15, Jun. 2019.

[8] Y.-T. Chan and K. Ho, "A simple and efficient estimator for hyperbolic location," IEEE Transactions on Signal Processing, vol. 42, no. 8, pp. 1905-1915, 1994.

[9] M. D. Gillette and H. F. Silverman, "A linear closed-form algorithm for source localization from time-differences of arrival," IEEE Signal Processing Letters, vol. 15, pp. 1-4, 2008.

[10] H. C. So, Y. T. Chan, and F. K. W. Chan, "Closed-form formulae for time-difference-of-arrival estimation," IEEE Transactions on Signal Processing, vol. 56, no. 6, pp. 2614-2620, 2008.

[11] D. Carevic, "Automatic estimation of multiple target positions and velocities using passive tdoa measurements of transients," IEEE Transactions on Signal Processing, vol. 55, no. 2, pp. 424-436, 2007.

[12] J. Schroeder, S. Galler, and K. Kyamakya, "A low-cost experimental ultra-wideband positioning system," in 2005 IEEE International Conference on Ultra-Wideband. IEEE, 2005, pp. 632-637.

[13] K. Yang, G. Wang, and Z.-Q. Luo, "Efficient convex relaxation methods for robust target localization by a sensor network using time differences of arrivals," IEEE Transactions on Signal Processing, vol. 57, no. 7, pp. 2775-2784, 2009.

[14] G. Wang, Y. Li, and N. Ansari, "A semidefinite relaxation method for source localization using TDOA and FDOA measurements," IEEE Transactions on Vehicular Technology, vol. 62, no. 2, pp. 853-862, 2012.

[15] T. Ballal and C. J. Bleakley, "Phase-difference ambiguity resolution for a single-frequency signal," IEEE Signal Processing Letters, vol. 15, pp. 853-856, 2008.

[16] H. Chen, T. Ballal, M. Saad, and T. Y. Al-Naffouri, "Angle-of-arrivalbased gesture recognition using ultrasonic multi-frequency signals," in 25th European Signal Processing Conference (EUSIPCO), 2017, pp. 16-20.

[17] Y. Chan, R. Hattin, and J. Plant, "The least squares estimation of time delay and its use in signal detection," IEEE Transactions on Acoustics, Speech, and Signal Processing, vol. 26, no. 3, pp. 217-222, 1978.

[18] P. V. Nikitin, R. Martinez, S. Ramamurthy, H. Leland, G. Spiess, and K. V. S. Rao, "Phase based spatial identification of UHF RFID tags," in IEEE International Conference on RFID (IEEE RFID), April 2010, pp. $102-109$.

[19] L. Qiu, Z. Huang, S. Zhang, C. Jing, H. Li, and S. Li, "Multifrequency phase difference of arrival range measurement: Principle, implementation, and evaluation," International Journal of Distributed Sensor Networks, vol. 11, no. 11, p. 715307, 2015.

[20] J. Kennedy, "Particle swarm optimization," Encyclopedia of machine learning, pp. 760-766, 2010.

[21] W. Jatmiko, K. Sekiyama, and T. Fukuda, "A pso-based mobile robot for odor source localization in dynamic advection-diffusion with obstacles environment: theory, simulation and measurement," IEEE Computational Intelligence Magazine, vol. 2, no. 2, pp. 37-51, 2007.

[22] R. V. Kulkarni and G. K. Venayagamoorthy, "Particle swarm optimization in wireless-sensor networks: A brief survey," IEEE Transactions on Systems, Man, and Cybernetics, Part C (Applications and Reviews), vol. 41, no. 2, pp. 262-267, 2010.

[23] P. Wu, S. Su, Z. Zuo, X. Guo, B. Sun, and X. Wen, "Time difference of arrival (TDoA) localization combining weighted least squares and firefly algorithm," Sensors, vol. 19, no. 11, p. 2554, 2019.

[24] M. R. Gholami, S. Gezici, and E. G. Strom, "A concave-convex procedure for TDOA based positioning," IEEE Communications Letters, vol. 17, no. 4, pp. 765-768, April 2013.

[25] T. Ballal and C. J. Bleakley, "Phase-difference ambiguity resolution for a single-frequency signal in the near-field using a receiver triplet," IEEE Transactions on Signal Processing, vol. 58, no. 11, pp. 5920-5926, Nov 2010.

[26] H. Chen, T. Ballal, N. Saeed, M.-S. Alouini, and T. Y. Al-Naffouri, "A Lower Bound for Joint TDOA-PDOA Localization (supplementary material)," https://github.com/chenhui07c8/Localization-algorithms, 2020. 